\title{
Gas-Surface Interaction Effect on Round Leading Edge Aerothermodynamics
}

\author{
Wilson F. N. Santos \\ National Institute for Space Research, Cachoeira Paulista, SP 12630-000, Brazil
}

Received on 17 November, 2006

\begin{abstract}
Effects of incomplete surface accommodation in rarefied gas flow have been studied by using the Direct Simulation Monte Carlo (DSMC) method in conjunction with the Cercignani-Lampis-Lord gas surface interaction model. The DSMC calculations examine differences in predictions of aerodynamic forces and heat transfer between full and partial surface accommodation for hypersonic flow past round leading edges at zero incidence. The aerodynamic performance of round leading edges is assessed by using the heat transfer rate, the total drag and the shock wave standoff distance. Twenty-five combinations of nose radius, normal and tangential accommodation coefficients were used in the simulation. For the flow conditions considered, the analysis showed that stagnation point heating, total drag and shock standoff distance are sensitive to changes on either the normal or tangential accommodation coefficient. The results substantiate that it becomes imperative to take surface accommodation into account in order to make accurate predictions of the aerodynamic forces on, and heat transfer rates to, bodies in rarefied hypersonic flow.
\end{abstract}

Keywords: Aerothermodynamics; Gas flow; Surfaces

\section{INTRODUCTION}

Supersonic and hypersonic flight vehicles are commonly designed and manufactured with blunt noses. A large nose radius helps to withstand, distribute, and dissipate the heat and pressure loadings that are often most extreme at the vehicle bow. At one hand, for the particular case of atmospheric entry and re-entry vehicles, high bluntness contributes to the drag production that is necessary to decelerate from suborbital to subsonic speed. On the other hand, supersonic and hypersonic cruise vehicles need low drag to efficiently maintain velocity, a requirement satisfied with a small but finite nose bluntness. In this scenario, the leading edge of the vehicle is one of the key issues concerning hypersonic configurations.

Certain configurations, such as hypersonic waveriders [1], are designed analytically with infinitely sharp leading edges for shock wave attachment in order to contain the highpressure air that produces useful lifting force. Nevertheless, these leading edges must then be blunted for heat transfer and manufacturing, with associated departure from ideal performance. An ongoing in the application of hypersonic waverider shapes to the design of realistic flight systems is therefore the concern that the predicted performance of analytically derived shapes, with infinitely sharp leading edges, will not be achieve when actual leading edges are blunted for heat transfer and manufacturing requirements. In this way, it has been generally assumed that a round leading edge, with constant radius of curvature near the stagnation point, is the appropriate blunting geometry.

Recently, considerable attention [2-6] has been given to the problem of calculating aerodynamic performance of hypersonic waverider vehicles for high-altitude/low-density applications. Nonetheless, in hypersonic flight at high altitudes, gas-surface interaction is the dominant physical process governing aerodynamic forces and heat transfer. The influence of the gas-surface interaction model on the flow parameters increases substantially as the gas rarefaction increases, and a correct choice of the model for calculating hypersonic rarefied flows plays an important role.

It has been known from experimental data that one can approximate gas-surface interactions on engineering surface with contamination from air and surface roughness by using the fully diffusion reflection model. Diffuse reflection occurs in such a manner that all previous directional history is erased, and the molecules are reflected equally in all directions. Nevertheless, according to molecular beam studies and direct measurements of accommodation, the diffuse reflection model is unrealistic, except for highly contaminated surface. The vehicle surface at high altitude will become gradually decontaminated, and it is likely that the reduction in accommodation will have significant effects on aerodynamic forces and heat transfer rates. As a result, molecules reflected from clean surface show lobular distribution in direction, which tends to approach the specular angle for very high energy and/or low angle of attack. Among the several phenomenological models that have been proposed to describe gas-surface interactions, that of Cercignani-Lampis-Lord (CLL) model [7] appears to be the most successful to handle such behavior. The CLL model incorporates independent accommodation coefficients for the normal and tangential velocity components. It produces physically realistic distributions of direction and energy of scattered molecules and provides a continuous spectrum of behavior from specular to diffuse reflection.

The study at hand investigates the differences in the aerothemodynamic quantities predicted with the CLL model, which is implemented into a DSMC code. For the idealized situation of two-dimensional rarefied hypersonic flow, calculations have been performed on round leading edges. Of particular interest are the behaviors of stagnation point heating, total drag and shock wave displacement.

\section{GAS-SURFACE INTERACTION MODEL}

The majority of gas dynamic problems includes the interaction of gas particles with the body surface. The influ- 
ence of the gas-surface interaction model on the flow properties increases substantially with increasing the gas rarefaction. Therefore, a correct choice of the model for calculating hypersonic rarefied flows is important.

Diffusion reflection with complete momentum and energy accommodation is most frequently used in DSMC method. In a diffuse reflection, the molecules are reflected equally in all directions usually with a complete thermal accommodation. The final velocity of the molecules is randomly assigned according to a half-range Maxwellian distribution determined by the wall temperature.

Specular reflection with zero accommodation or complementary fractions of diffuse and specular reflection are also often used in DSMC method. The combination of diffuse reflection with specular reflection is called Maxwell model. Generally, the Maxwell model is constructed on the assumption that a fraction $(1-f)$ of the molecules is reflected from the surface in a specular fashion, while the fraction $f$ is re-emitted diffusely. The parameter $f$ in the Maxwell model is identified by the tangential accommodation coefficient $\sigma_{t}$.

The popularity of the Maxwell model is explained apparently by its simplicity and by the fact that it satisfies reciprocity, i.e., the principle of detailed balance. A phenomenological model that also satisfies the reciprocity conditions and has demonstrated improvement over the Maxwell model has been proposed by Cercignani and Lampis [8], known as CL model. This model is based on the definition of two parameters $\alpha_{n}$ and $\sigma_{t}$ that represent the accommodation coefficient for the kinetic energy associated with the normal component of velocity, and the parallel momentum accommodation, respectively. They are given by the following equations,

$$
\begin{gathered}
\alpha_{n}=\frac{e_{i}-e_{r}}{e_{i}-e_{w}} \\
\sigma_{t}=\frac{\tau_{i}-\tau_{r}}{\tau_{i}}
\end{gathered}
$$

where $e$ and $\tau$ refer to the average kinetic energy for the normal component of velocity, and momentum flux acting tangential to the surface, respectively; subscripts $i$ and $r$ stand for the incident and reflected components, and $w$ refers to the component that would be produced by a diffuse reflection at the temperature of the surface.

The CL model provides a continuous spectrum of behavior from specular reflection at one end to diffuse reflection with complete energy accommodation at the other, and produces physically realistic distributions of direction and energy reemitted molecules.

The probability distribution function for the component of velocity normal to the surface is given by,

$$
\begin{gathered}
P_{n}\left(v_{n}^{\prime} \mid v_{n}\right)=\frac{2 v_{n}^{\prime}}{\alpha_{n}} I_{0}\left(2 \sqrt{1-\alpha_{n}} \frac{v_{n} v_{n}^{\prime}}{\alpha_{n}}\right) \times \\
\exp \left[-\frac{v_{n}^{\prime 2}+\left(1-\alpha_{n}\right) v_{n}^{2}}{\alpha_{n}}\right]
\end{gathered}
$$

where $I_{0}$ is the modified Bessel function, $v_{n}^{\prime}$ and $v_{n}$ are the incident and reflect velocity components normalized by the most probable molecular speed at the surface temperature, and defined by $\sqrt{2 k T_{w} / m}$.

The probability distribution function for the component of velocity parallel to the surface is given by,

$$
\begin{aligned}
P_{t}\left(\mathbf{v}_{t}^{\prime} \mid \mathbf{v}_{t}\right)= & \frac{1}{\sqrt{\pi \sigma_{t}\left(2-\sigma_{t}\right)}} \times \\
& \exp \left\{-\frac{\left.v_{t}^{\prime}-\left(1-\sigma_{t}\right) v_{t}\right]^{2}}{\pi \sigma_{t}\left(2-\sigma_{t}\right)}\right\}
\end{aligned}
$$

where $\mathbf{v}_{t}^{\prime}$ and $\mathbf{v}_{t}$ are the incident and reflect tangential velocity vector also normalized by the most probable molecular speed at the surface temperature.

Recently, Lord [7] has presented the implementation of the CL model into the DSMC method, and presented a simple method for generating random sample velocities from Eqs. (3) and (4). The DSMC method with Lord's implementation is referred as the Cercignani-Lampis-Lord (CLL) model. Fig. 1 displays a schematic comparison of the Maxwell reflection model and the CLL reflection model.

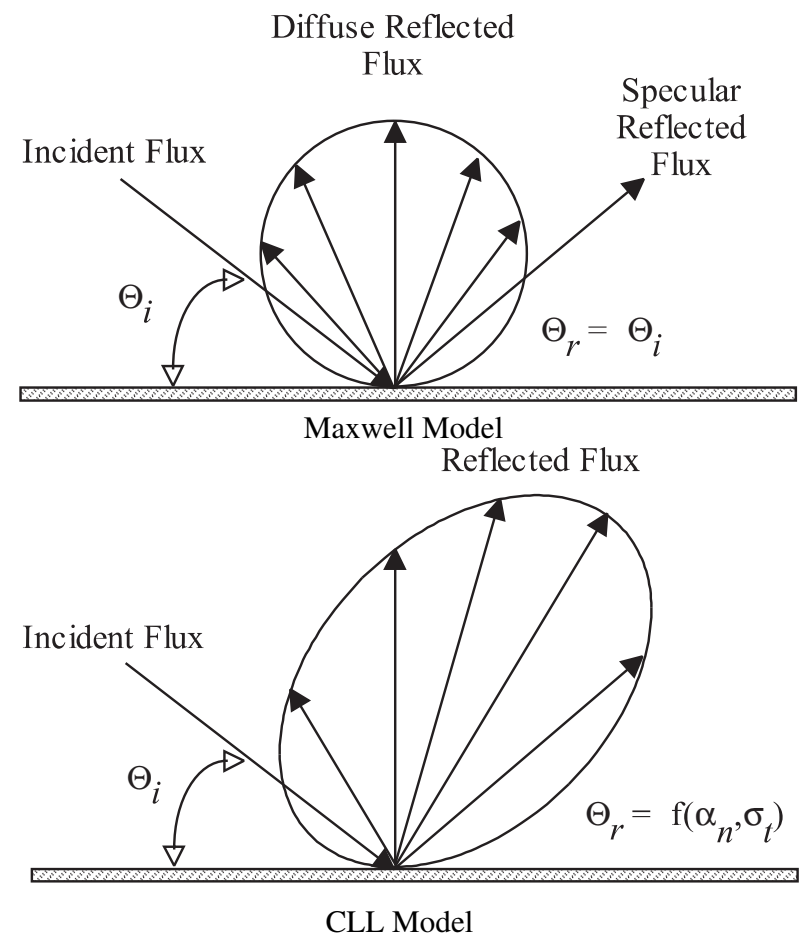

FIG. 1: Drawing illustrating the Maxwell reflection model and the CLL reflection model.

It should be emphasized that the accommodation coefficient may be considered as a term that accounts for the inability of the impinging molecules to adjust themselves to the body surface during the time they are in contact with the body surface. In addition, if defined rigorously, it would have different values for translational, rotational and vibrational energies. Nevertheless, experimental data have shown that the accommodation coefficients were numerically identical for rotational and 
translational energies. The vibrational components of the internal energy require much more time to adjust to new values than do the rotational and translational components. In this connection, Lord [9-11] has also been extended the CL model for simulating the accommodation of vibrational energy of a diatomic molecule.

In order to simulate the partial surface accommodation, the Cercignani-Lampis-Lord (CLL) model [7] was implemented into this DSMC calculation. The CLL model is derived assuming that there is no coupling between the normal and tangential momentum components. The two adjustable parameters appearing in the CLL model are the normal component of translational energy $\alpha_{n}$ and the tangential component of momentum $\sigma_{t}$. However, in the implementation of the CLL model in the DSMC method, Bird [12] has shown that it is equivalent to specify the normal $\alpha_{n}$ and tangential $\alpha_{t}$ components of translational energy, since $\alpha_{t}=\sigma_{t}\left(2-\sigma_{t}\right)$, and thus that $\sigma_{t}<\alpha_{t}$, assuming that $\sigma_{t}$ lies between 0 and 1 .

In the present account, $\alpha_{n}$ and $\sigma_{t}$ are used as being the two adjustable parameters. It is important to mention that in the CLL model the accommodation of internal energy is allowed to be independent of the translational accommodation.

Before proceeding with the analysis, it is important to mention that many studies have been made using both the CL and the CLL models to analyze the behavior of the reflected molecules. Of particular interest are the application of the CL model described in Ref.[13,14] and those on CLL model discussed in Ref.[15,16].

\section{LEADING EDGE GEOMETRY DEFINITION}

The round leading edges are modeled by assuming a sharp leading edge of half angle $\theta$ with a reference circular cylinder of radius $R$ inscribed tangent to the wedge. The round leading edges are inscribed between the wedge and the cylinder. The circular cylinder diameter provides a reference for the amount of blunting desired on the leading edges. It was assumed a leading edge half angle of 10 degrees and a reference circular cylinder diameter of $10^{-2} \mathrm{~m}$. In addition to the reference circular cylinder, four more circular cylinders with different nose radii were chosen for round leading edges. The dimensionless nose radius $R_{N} / \lambda_{\infty}$ for the four bodies are $0.02,0.1,1$ and 2 , where $\lambda_{\infty}$ is the freestream mean free path. Figure 2 illustrates the construction for the round leading edges investigated.

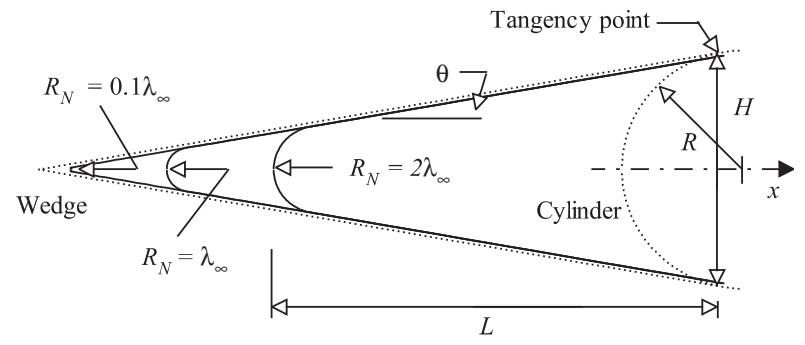

FIG. 2: Drawing illustrating the leading edge geometries.

\section{COMPUTATIONAL METHOD AND PROCEDURE}

It is well known that the Direct Simulation Monte Carlo (DSMC) method, introduced by Bird [12] in the 1960's, has become a reliable and efficient kinetic approach for modeling rarefied gas flows. Typical applications include high altitude rockets plumes, microelectromechanical systems (MEMS) devices, spacecraft propulsion and contamination, low-pressure plasma material-processing reactors, and reentry vehicles. Although these applications encompass a wide range of spatial and temporal scales, they are united by the same underlying physics of moderate or high Knudsen number flows.

The Knudsen number $K n$ is de ratio of the gas mean free path $\lambda$ to the characteristic length scale $l$ of the problem. It is generally accepted that the rarefied transition flow regime lies in the range of $0.01<K n<10$. The transition regime is the category of flow that falls between the continuum regime, where the Navier-Stokes equations are valid, and the free molecular regime, which is the limit of infinite Knudsen number. Currently, mathematical modeling of flows in the transition flow regime is supposed to be based on the solution of the Boltzmann equation [17] or on the DSMC method [12].

The DSMC method employs thousands or millions of representative molecules in order to reproduce the behavior of a far larger number of real molecules within the flow. The strategy of the method is to directly track the molecular trajectory and status based on the collision mechanics to model molecules in a computer and then obtain physical quantities of interest through statistical sampling.

The fundamental assumption employed in the DSMC method is that the movement of the molecules in a dilute gas can be decoupled from the collisions between the molecules if a sufficient small time step is employed. The time step has to be selected in such a way that the distance traveled by a molecule during each time step is smaller than the mean free time $[18,19]$ between collision of the real gas.

Collisions in the present DSMC code are modeled by using the variable hard sphere (VHS) molecular model [20] and the no time counter (NTC) collision sampling technique [21]. Repartition energy among internal and translational modes is controlled by the Borgnakke-Larsen statistical model [22]. Simulations are performed using a non-reacting gas model for a constant freestream gas composition consisting of $76.3 \%$ of $\mathrm{N}_{2}$ and $23.7 \%$ of $\mathrm{O}_{2}$. Energy exchanges between the translational and internal modes, rotational and vibrational, are considered. Relaxation collision numbers of 5 and 50 were used for the calculations of rotation and vibration, respectively.

In the DSMC method, the physical space is divided into a certain number of cells and each cell is also divided into subcells. The physical space network is used to facilitate the choice of molecules for collisions and for the sampling of the macroscopic flow properties such as temperature, pressure, etc. In the DSMC algorithm, the linear dimensions of the cells should be small in comparison with the scale length of the macroscopic flow gradients normal to the streamwise directions, which means that the cell dimensions should be of the order of or smaller than the local mean free path [23,24]. 


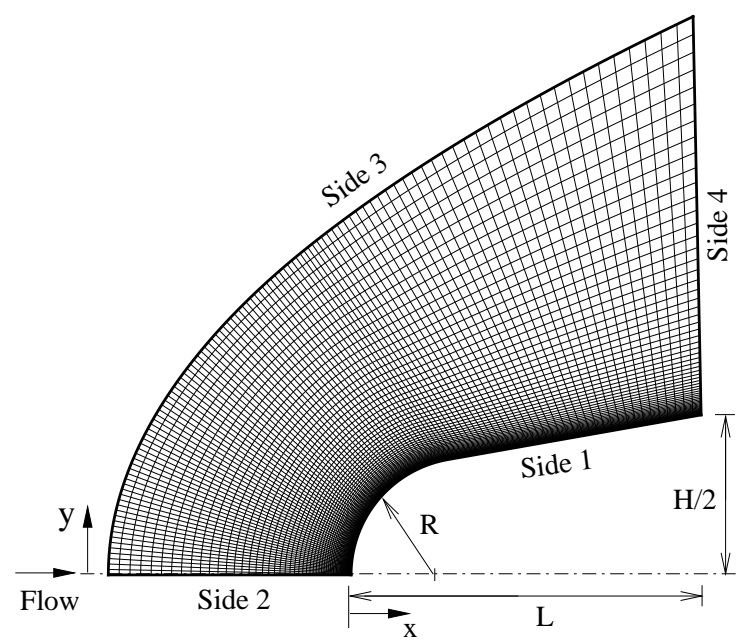

FIG. 3: Schematic view of the computational domain.

The computational domain used for the calculation is made large enough so that body disturbances do not reach the upstream and side boundaries, where freestream conditions are specified. A schematic view of the computational domain is depicted in Fig. 3. Based on this figure, side 1 is defined by the body surface. Reflection with incomplete surface accommodation is the condition applied to this side. Advantage of the flow symmetry is taken into account, and molecular simulation is applied to one-half of a full configuration. Thus, side 2 is a plane of symmetry. In such a boundary, all flow gradients normal to the plane are zero. At the molecular level, this plane is equivalent to a specular reflecting boundary. Side 3 is the freestream side through which simulated molecules enter and exit. Finally, the flow at the downstream outflow boundary, side 4 , is predominantly supersonic and vacuum condition is specified [25]. At this boundary, simulated molecules can only exit.

The numerical accuracy in DSMC method depends on the cell size chosen, on the time step as well as on the number of particles per computational cell. These effects were investigated in order to determine the number of cells and the number of particles required to achieve grid independence solutions. Grid independence was tested by running the calculations with half and double the number of cells, along the body surface and off-body surface, and then compared to a standard grid. Solutions were near identical for all grids used and were considered fully grid independent. Details are given in the appendix.

No attempt has been made to compare the results of the present calculations with such experimental results as exist. Most of these have been obtained in wind-tunnel environments where no control of surface condition is possible and surface would be expected to be highly contaminated and to exhibit virtually complete accommodation. Moreover, the relative few experiments in which effects of partial accommodation appear have all used helium rather that air.

\section{FREESTREAM AND FLOW CONDITIONS}

The flow conditions represent those experienced by a spacecraft at an altitude of $70 \mathrm{~km}$. This altitude is associated with the transition flow regime, which is characterized by the overall Knudsen number of the order of or larger than $10^{-2}$. The freestream conditions are summarized in Table I, and the gas properties [12] are shown in Table II.

TABLE I: Freestream and flow conditions

\begin{tabular}{lll}
\hline \hline Parameter & Value & Unit \\
\hline Altitude & 70 & $\mathrm{~km}$ \\
Temperature $\left(T_{\infty}\right)$ & 220.0 & $\mathrm{~K}$ \\
Pressure $\left(p_{\infty}\right)$ & 5.582 & $\mathrm{~N} / \mathrm{m}^{2}$ \\
Density $\left(\rho_{\infty}\right)$ & $8.753 \times 10^{-5}$ & $\mathrm{~kg} / \mathrm{m}^{3}$ \\
Viscosity $\left(\mu_{\infty}\right)$ & $1.455 \times 10^{-5}$ & $\mathrm{Ns} / \mathrm{m}^{2}$ \\
Number density $\left(n_{\infty}\right)$ & $1.8209 \times 10^{21}$ & $\mathrm{~m}^{-3}$ \\
Mean free path $\left(\lambda_{\infty}\right)$ & $9.03 \times 10^{-4}$ & $\mathrm{~m}$ \\
\hline \hline
\end{tabular}

TABLE II: Gas properties

\begin{tabular}{llll}
\hline \hline Parameter & $\mathrm{O}_{2}$ & $N_{2}$ & Unit \\
\hline Molecular mass & $5.312 \times 10^{-26}$ & $4.65 \times 10^{-26}$ & $\mathrm{~kg}$ \\
Molecular diameter & $4.010 \times 10^{-10}$ & $4.11 \times 10^{-10}$ & $\mathrm{~m}$ \\
Mole fraction & 0.237 & 0.763 & \\
Viscosity index & 0.77 & 0.74 & \\
\hline \hline
\end{tabular}

The freestream velocity $V_{\infty}$ is assumed to be constant at $3.56 \mathrm{~km} / \mathrm{s}$, which correspond to a freestream Mach number $M_{\infty}$ of 12 . The translational and vibrational temperatures in the freestream are in equilibrium at $220 \mathrm{~K}$, and the leadingedge surface has a temperature $T_{w}$ of $880 \mathrm{~K}$ for all cases considered. This temperature is chosen to be representative of the surface temperature near the stagnation point and is assumed to be uniform over the bodies. It is important to mention that the surface temperature is low compared to the stagnation temperature of the air. This assumption seems reasonable since practical surface material will probably be destroyed if surface temperature is allowed to approach stagnation temperature.

By assuming the nose diameter as the characteristic length, the overall Knudsen number $K n_{D}$ corresponds to $25,5,0.5$, 0.25 and 0.09 for nose radius $R_{N} / \lambda_{\infty}$ of $0.02,0.1,1,2$ and 5.5 , respectively. The Reynolds number per unit of meter is $R e_{\infty}=21416.3$, also based on conditions in the undisturbed stream.

In order to simulate the incomplete surface accommodation, the CLL model implemented into the DSMC code considered only the normal and tangential accommodation coefficients. The internal energy accommodation was kept equal to one for all calculations presented in this work. Hence, 
$\alpha_{n}$ and $\sigma_{t}$ are used as being the two adjustable parameters. The DSMC calculations were performed independently for three distinct numerical values for $\alpha_{n}$ and $\sigma_{t}: 0.5,0.75$ and 1. Therefore, when $\alpha_{n}$ is equal to 0.5 or $0.75, \sigma_{t}$ is set equal to 1 , and vice-versa. It is important to mention that $\alpha_{n}$ and $\sigma_{t}$ equal to 1 represent the diffusion reflection case.

\section{COMPUTATIONAL RESULTS AND DISCUSSION}

The aerodynamic performance of the round leading edges is assessed by using the heat transfer rate, the total drag and the shock wave standoff distance. In this way, the purpose of this section is to discuss differences in these properties due to variations on the surface accommodation coefficients of these bodies with different nose radius.

\section{A. Heat Transfer Coefficient}

Energy may be added to or subtracted from the body surface by three distinct processes: (1) molecular energy transport to and from the body surface, (2) radiant energy transport to and from the body surface, and (3) energy added to or removed from the surface by processes occurring within the body. By considering process (1), the heat flux $q_{w}$ to the body surface is calculated by the net energy flux of the molecules impinging on the surface. A flux is regarded as positive if it is directed toward the surface. The net heat flux $q_{w}$ is related to the sum of the translational, rotational and vibrational energies of both incident and reflected molecules as defined by,

$$
\begin{array}{r}
q_{w}=q_{i}+q_{r}=\sum_{j=1}^{N}\left[\frac{1}{2} m_{j} c_{j}^{2}+e_{R j}+e_{V j}\right]_{i}+ \\
\sum_{j=1}^{N}\left[\frac{1}{2} m_{j} c_{j}^{2}+e_{R j}+e_{V j}\right]_{r}
\end{array}
$$

where $N$ is the number of molecules colliding with the surface by unit time and unit area, $m$ is the mass of the molecules, $c$ is the velocity of the molecules, $e_{R}$ and $e_{V}$ stand for the rotational and vibrational energies, respectively, and subscripts $i$ and $r$ refer to incident and reflect molecules. by,

In the following, the heat transfer coefficient $C_{h}$ is defined

$$
C_{h}=\frac{q_{w}}{\rho_{\infty} V_{\infty}^{3} / 2}
$$

Distributions of the heat transfer coefficient $C_{h}$ along the round leading edge surface are illustrated as a function of the accommodation coefficients in Figs. 4(a-c) for nose radius $R_{N} / \lambda_{\infty}$ of $0.02,1$ and 5.5 , respectively. In this set of figures, $S$ is the arc length $s$ normalized by the freestream mean free path $\lambda_{\infty}$ measured from the stagnation point. The heat transfer coefficient $C_{h}$ for $R_{N} / \lambda_{\infty}$ of 0.1 and 2 is intermediate to the cases shown in this set of figures, therefore they will not be presented. Once again, it is important to emphasize that the accommodation coefficient effects are investigated in an independent way. For instance, the curve corresponding to $\alpha_{n}=$ 0.5 or 0.75 in Figs. 4(a-c) represents the situation of $\alpha_{n}=0.5$ or 0.75 with $\sigma_{t}=1$. Similarly, the curve corresponding to $\sigma_{t}$ $=0.5$ or 0.75 represents the case $\sigma_{t}=0.5$ or 0.75 with $\alpha_{n}=1$.
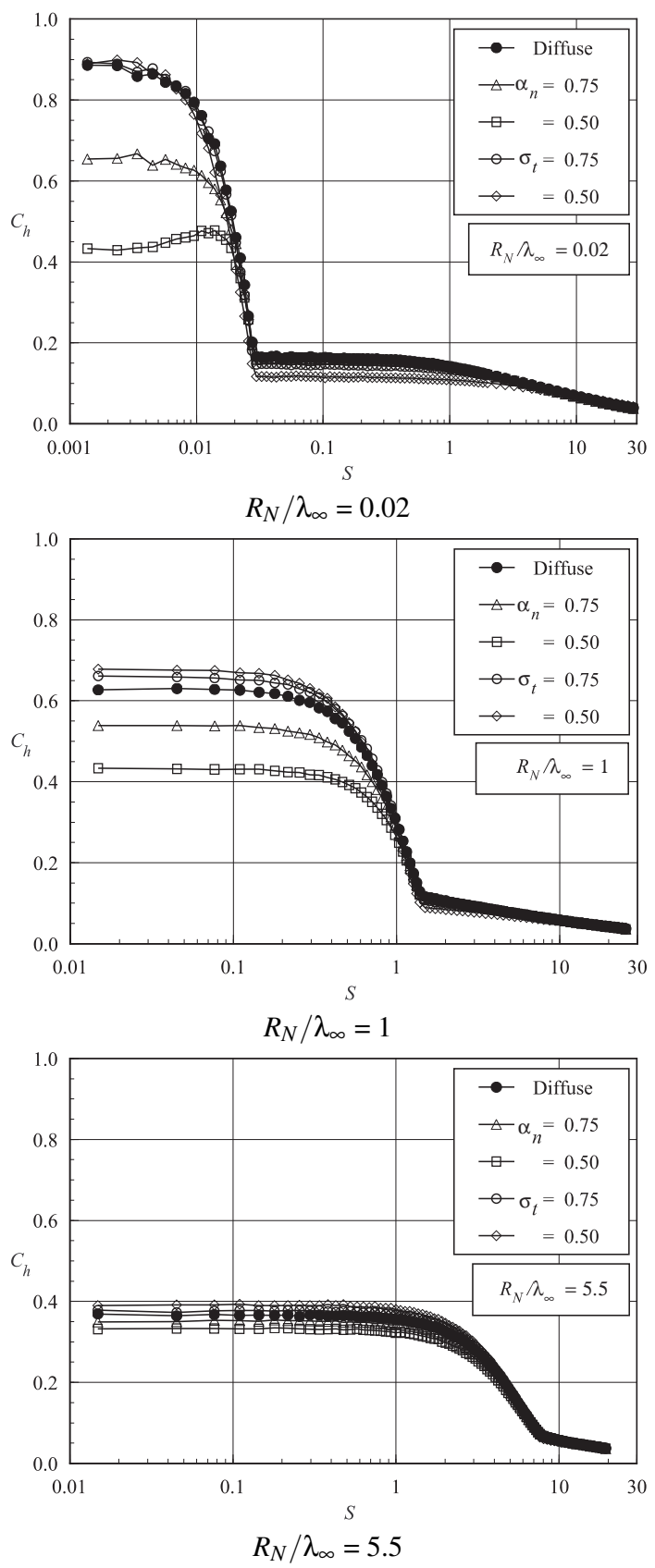

FIG. 4: Distributions of the heat transfer coefficient $C_{h}$ along the body surface as a function of the accommodation coefficient for round leading edges with $R_{N} / \lambda_{\infty}$ of (a) 0.02 , (b) 1 and (c) 5.5 .

It is apparent from Figs. 4(a-c) that the heat transfer coefficient is sensitive not only to the normal and tangential accommodation coefficients but also to the nose radius. In general, the heat transfer coefficient presents the maximum value at 
the stagnation point and drops off sharply along the cylindrically blunt portion up to the cylinder/wedge junction. It is noted from this set of figures that the heat transfer coefficient decreases by a reduction in the normal accommodation coefficient and it increases with reducing the tangential accommodation coefficient. Moreover, either the normal or tangential accommodation coefficient affects the leading edges in a different way as the nose radius $R_{N} / \lambda_{\infty}$ is reduced from 5.5 to 0.02 . In addition to that, the heat transfer coefficient at the stagnation region decreases with increasing the nose radius. This behavior seems to be in agreement with the continuum predictions for blunt body in that the heat flux scales inversely with the square root of the nose radius. As expected, by reducing the nose radius the leading edge becomes sharper and approaches the wedge leading edge as shown in Fig. 2.

Effects of both the incomplete surface accommodation and the nose radius on the heat transfer coefficient $C_{h}$ can also be seen in a different way by displaying the results as a function of the body slope angle $\theta$. Figures 5(a-c) depict the heat transfer coefficient on the cylindrically portion of the leading edges as a function of the body slope angle for diffuse case, $\alpha_{n}$ of 0.5 (with $\sigma_{t}=1$ ) and $\sigma_{t}$ of 0.5 (with $\alpha_{n}=1$ ), respectively. For comparison purpose, Fig. 5(a) presents the heat transfer coefficient $C_{h}$ by assuming free-molecule (FM) flow [12].

Referring to Fig. 5(a), it is noted that the heat transfer coefficient approaches the free molecular limit $\left(C_{h o}=0.915\right)$ in the cylindrically portion of the round leading edge with reducing the nose radius. It is clearly seen in Figs. 5(a-c) that the heat transfer coefficient profiles are preserved for the majority of the cases investigated. In general the heat transfer coefficient presents the maximum value at the stagnation region and decreases along the cylindrically portion of the leading edges. However, for cases with $R_{N} / \lambda_{\infty}$ of 0.02 and 0.1 , and $\alpha_{n}$ of 0.5 (see Fig. 5(b)), the maximum point moves from the stagnation point, which corresponds to station $\theta=90$ degrees, to station around $\theta=50$ degrees. In an effort to emphasize this behavior, the incident and reflect heat flux contributions to the net heat transfer coefficient are shown separately.

The incident $C_{h i}$ and reflected $C_{h r}$ heat transfer coefficient distributions along the cylindrically portion of the round leading edges for $R_{N} / \lambda_{\infty}$ of $0.02,0.1$ and 5.5 are illustrated in Figs. 6(a-c), respectively. In this set of diagrams, full and empty symbols stand for the incident and reflected heat transfer coefficients, respectively. It is observed from these figures that the incident heat transfer coefficients are not affected by changes in the normal accommodation coefficient. In contrast, the reflected heat flux increases with reducing the normal accommodation coefficient. In addition, the reflected heat flux is sensitive to the leading edge shape in that it presents a larger value at the stagnation region for sharp leading edge than that for blunt leading edge.

At this point, it seems important to quantify the heat transfer at the stagnation point. The heat transfer coefficient at the stagnation point $C_{h o}$ is displayed in Table III for the range of nose radius investigated. These values were obtained by a curve fitting process performed over the curves shown in Figs. 5(a-c).

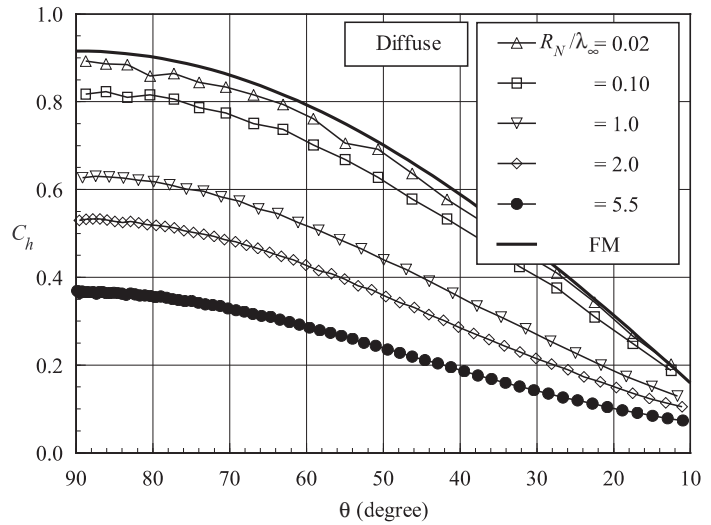

Diffuse reflection

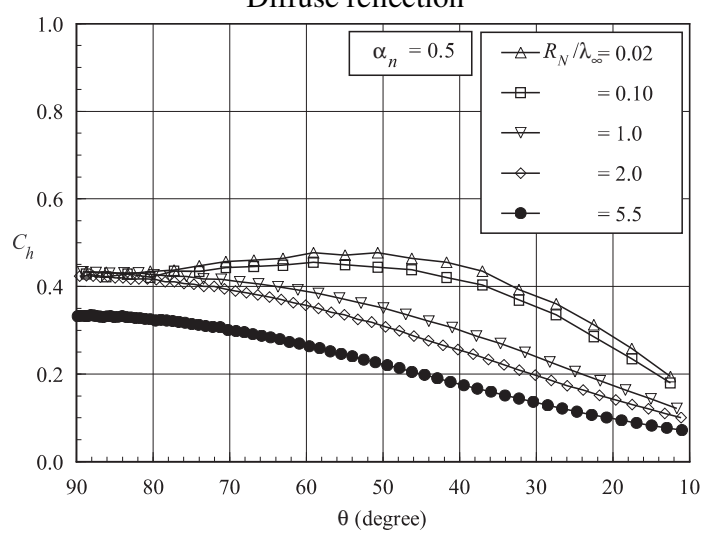

$\alpha_{n}=0.5$ and $\sigma_{t}=1$

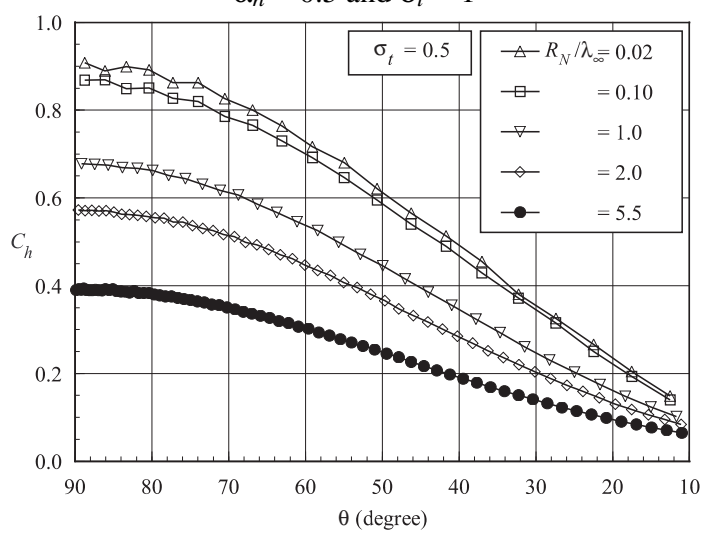

$\alpha_{n}=1$ and $\sigma_{t}=0.5$

FIG. 5: Distribution of the heat transfer coefficient $C_{h}$ along the cylindrically portion of the round leading edges by considering (a) diffuse reflection, (b) $\alpha_{n}=0.5$ and $\sigma_{t}=1$, and (c) $\alpha_{n}=1$ and $\sigma_{t}=$ 0.5 .

\section{B. Total Drag Coefficient}

The total drag coefficient is defined as being,

$$
C_{d}=\frac{D}{\rho_{\infty} V_{\infty}^{2} H / 2}
$$

where $D$ is the resultant force acting on the body surface, and $H$ is the height at the matching point common to the leading edges (see Fig. 2). 


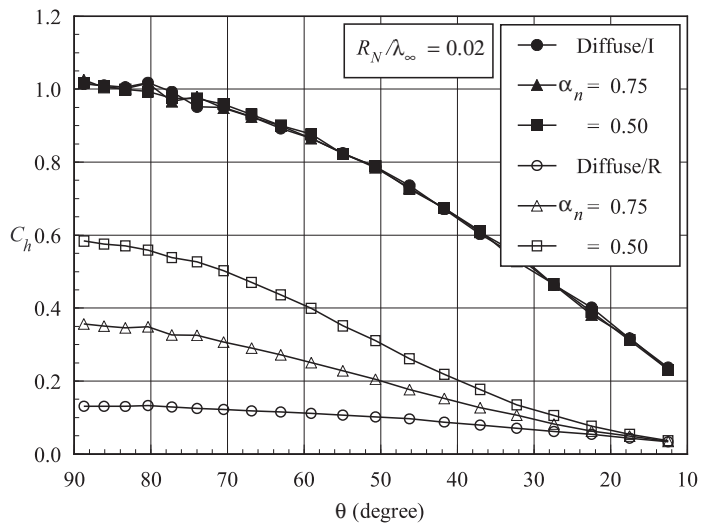

$R_{N} / \lambda_{\infty}=0.02$

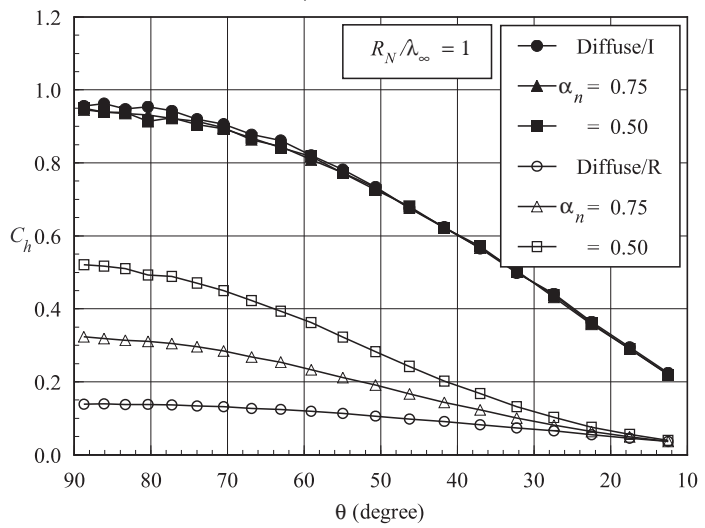

$R_{N} / \lambda_{\infty}=1$

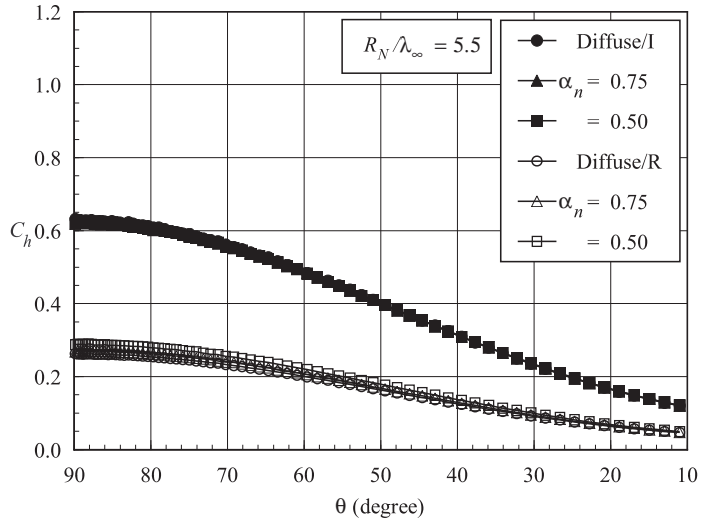

$R_{N} / \lambda_{\infty}=5.5$

FIG. 6: Distribution of the incident and reflected contributions of the heat transfer coefficient $C_{h}$ along the cylindrically portion of the round leading edges with $R_{N} / \lambda_{\infty}$ of (a) 0.02 , (b) 1 and (c) 5.5 .

The drag $D$ on a surface in a gas flow results from the interchange of momentum between the surface and the molecules colliding with the surface. The resultant force acting on the body surface is obtained by the integration of the pressure $p_{w}$ and shear stress $\tau_{w}$ distributions along the body surface. In this connection, $p_{w}$ and $\tau_{w}$ distributions were considered from the nose of the leading edge to the station $L$ (see Fig. 2), which corresponds to the tangent point common to all of the body shapes. The total drag presented in this section was obtained by assuming the shapes acting as leading edges. As a result,
TABLE III: Heat transfer coefficient at the stagnation point $C_{h o}$ for round leading edges.

\begin{tabular}{cccccc}
\hline \hline$R_{N} / \lambda_{\infty}$ & 0.02 & 0.1 & 1 & 2 & 5.5 \\
\hline Diffuse & 0.883 & 0.824 & 0.630 & 0.532 & 0.366 \\
$\alpha_{n}=0.75, \sigma_{t}=1$ & 0.660 & 0.662 & 0.537 & 0.484 & 0.352 \\
$\alpha_{n}=0.50, \sigma_{t}=1$ & 0.436 & 0.433 & 0.429 & 0.424 & 0.333 \\
$\alpha_{n}=1, \sigma_{t}=0.75$ & 0.894 & 0.859 & 0.660 & 0.553 & 0.377 \\
$\alpha_{n}=1, \sigma_{t}=0.50$ & 0.904 & 0.868 & 0.676 & 0.571 & 0.391 \\
\hline
\end{tabular}

no base pressure effects were taken into account on the calculations.

Before presenting the results for the total drag coefficient acting on the round leading edges, it proves helpful to visualize the manner in which the two accommodation coefficients as well as the nose radius affect pressure $p_{w}$ and shear stress $\tau_{w}$ distributions along the body surfaces.

The pressure $p_{w}$ on the body surface is calculated by the sum of the normal momentum fluxes of both incident and reflected molecules at each time step as follows,

$$
p_{w}=p_{i}+p_{r}=\sum_{j=1}^{N}\left\{\left[m_{j} c_{\eta_{j}}\right]_{i}+\left[m_{j} c_{\eta j}\right]_{r}\right\}
$$

where $c_{\eta}$ is the normal velocity component of the molecules colliding with the body surface.

The influence on wall pressure due to variations on the nose radius and on the surface accommodation coefficient is demonstrated in Fig. 7 in terms of the pressure coefficient $C_{p}$ defined by the following equation,

$$
C_{p}=\frac{p_{w}-p_{\infty}}{\rho_{\infty} V_{\infty}^{2} / 2}
$$

The variation of the pressure coefficient $C_{p}$ caused by changes on the surface accommodation coefficient is demonstrated in Figs. 7(a-c) for $R_{N} / \lambda_{\infty}$ of $0.02,0.1$ and 5.5, respectively. According to Figs. $7(\mathrm{a}-\mathrm{c})$, it is seen that the pressure coefficient $C_{p}$ follows the same trend as that presented by the heat transfer coefficient $C_{h}$ in that it presents the maximum value at the stagnation point and decreases fast in the cylindrically blunt portion of the leading edges.

It is also verified that the pressure coefficient $C_{p}$ in the cylindrically blunt portion is one order of magnitude higher than the pressure coefficient in the wedge portion of the leading edge. At one hand, Figs. 7(a-c) demonstrate that the pressure coefficient increases significantly at the vicinity of the stagnation point for sharp leading edges with reducing the normal accommodation coefficient $\alpha_{n}$. On the other hand, no appreciable changes are observed for those leading edges representing blunt leading edges. Consequently, the pressure coefficient is a sensitive function of the nose radius when the normal accommodation coefficient $\alpha_{n}$ is reduced from 1 to 0.5 . One possible reason for this higher surface pressure 
might be that the molecules that are reflected upstream have a high kinetic energy with the partial accommodation model and, thus, when they re-collide with the surface, they will impart a greater normal moment transfer. Moreover, it may also be recognized from Figs. 7(a-c) that the pressure coefficient is insensitive to changes on the tangential accommodation coefficient $\sigma_{t}$.

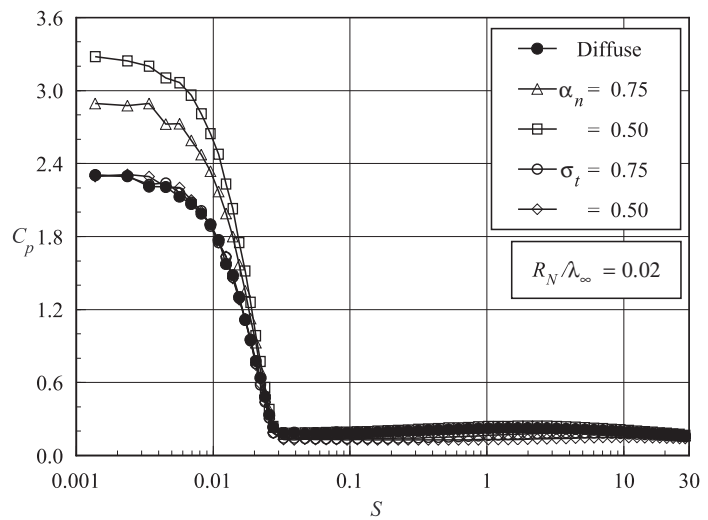

$R_{N} / \lambda_{\infty}=0.02$

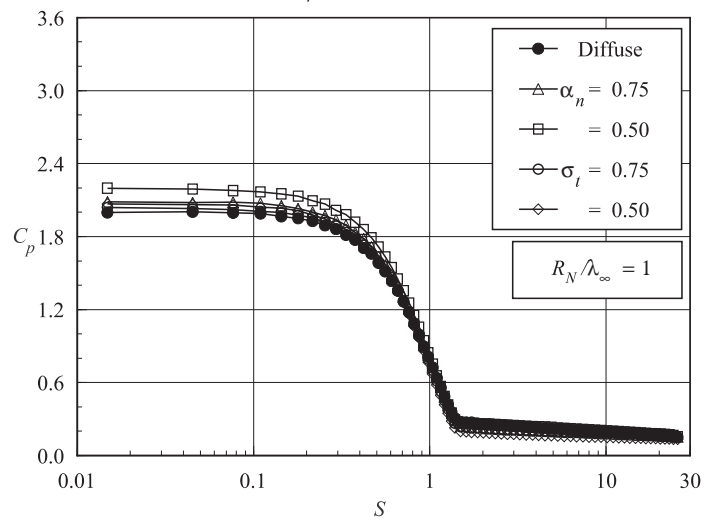

$R_{N} / \lambda_{\infty}=1$

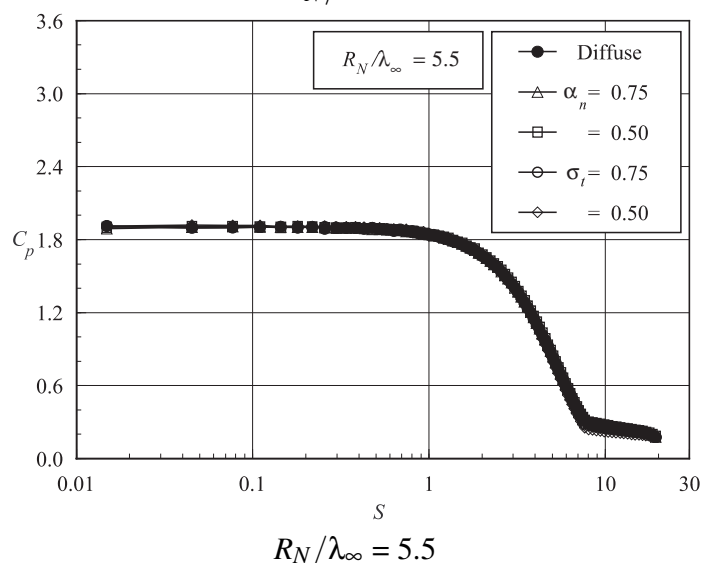

FIG. 7: Distributions of pressure coefficient $C_{p}$ along the body surface as a function of the accommodation coefficient for round leading edges with $R_{N} / \lambda_{\infty}$ of (a) 0.02 , (b) 1 and (c) 5.5 .

The shear stress $\tau_{w}$ on the body surface is calculated by the sum of the tangential momentum fluxes of both incident and reflected molecules impinging on the surface at each time step by the following expression,

$$
\tau_{w}=\tau_{i}+\tau_{r}=\sum_{j=1}^{N}\left\{\left[m_{j} c \xi_{j}\right]_{i}+\left[m_{j} c \xi_{j}\right]_{r}\right\}
$$

where $c_{\xi}$ is the tangential velocity component of the molecules colliding with the body surface.

The impact on wall shear stress due to variations on the nose radius and on the surface accommodation coefficient is displayed in Fig. 8 in terms of the skin friction coefficient $C_{f}$ defined as follows,

$$
C_{f}=\frac{\tau_{w}}{\rho_{\infty} V_{\infty}^{2} / 2}
$$

It is worthwhile to note that for the special case of diffuse reflection, $\alpha_{n}$ and $\sigma_{t}$ equal to 1 , the reflected molecules have a tangential moment equal to zero, since the molecules essentially lose, on average, their tangential velocity components. In this fashion, the contribution of the reflected tangential momentum flux $\tau_{r}$ that appears in Eq. (10) is equal to zero. Nevertheless, for incomplete surface accommodation, the reflected tangential momentum flux $\tau_{r}$ contributes to the skin friction coefficient.

The dependence of the skin friction coefficient $C_{f}$ attributed to variations on the surface accommodation coefficient is depicted in Figs. 8(a-c) for $R_{N} / \lambda_{\infty}$ of $0.02,0.1$ and 5.5, respectively. Based on this set of figures, the skin friction coefficient $C_{f}$ increases from zero at the stagnation point to a maximum value that is still located in the cylindrically blunt portion of the leading edges, and decreases downstream along the body surface. It is also seen that the skin friction coefficient presents an opposite behavior from that of pressure coefficient in the sense that it decreases with reducing the tangential accommodation coefficient $\sigma_{t}$. Furthermore, no changes are observed in the skin friction coefficient for a reduction on the normal accommodation coefficient $\alpha_{n}$ from 1 to 0.5 . Also of great significance is the skin friction changes on the afterbody surface with diminishing the tangential accommodation coefficient $\sigma_{t}$, in contrast to the pressure coefficient behavior.

The differences in the pressure $C_{p}$ and in the skin friction $C_{f}$ coefficients observed for variations on the normal $\alpha_{n}$ and tangential $\sigma_{t}$ accommodation coefficients as well as on the nose radius $R_{N}$ have been presented in Figs. 7 and 8 . In what follows, it becomes instructive to present the results for the total drag coefficient $C_{d}$, obtained by the integration of the pressure $p_{w}$ and shear stress $\tau_{w}$ distributions along the body surface.

The impact of partial accommodation coefficients $\alpha_{n}$ and $\sigma_{t}$ on the total drag coefficient $C_{d}$ is demonstrated in Figs. 9(a-c) for nose radius $R_{N} / \lambda_{\infty}$ of $0.02,1$ and 5.5 , respectively. With respect to this set of plots, it is observed that as the leading edge becomes blunt the contribution of the pressure drag $C_{p d}$ to the total drag $C_{d}$ increases and the contribution of the skin friction drag $C_{f d}$ decreases. For the sharpest round leading edge investigated, nose radius $R_{N} / \lambda_{\infty}$ of 0.02 , the major contribution to the total drag coefficient is attributed to the skin friction coefficient, a characteristic observed in a sharp leading edge. In contrast, for the bluntest case, nose radius $R_{N} / \lambda_{\infty}$ 


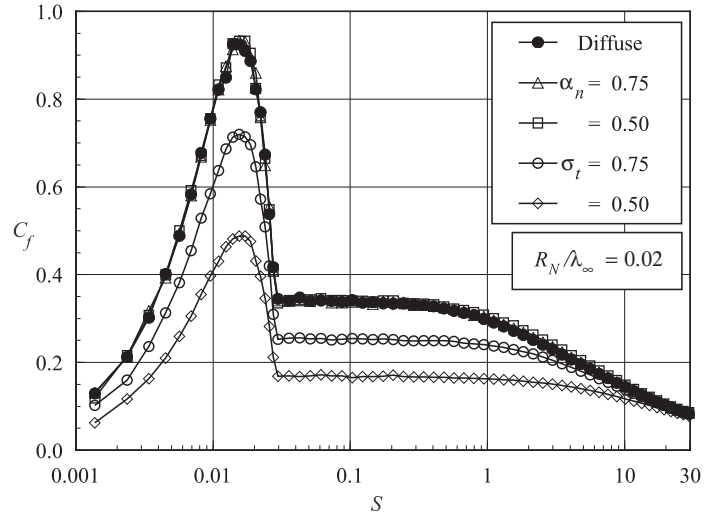

$R_{N} / \lambda_{\infty}=0.02$

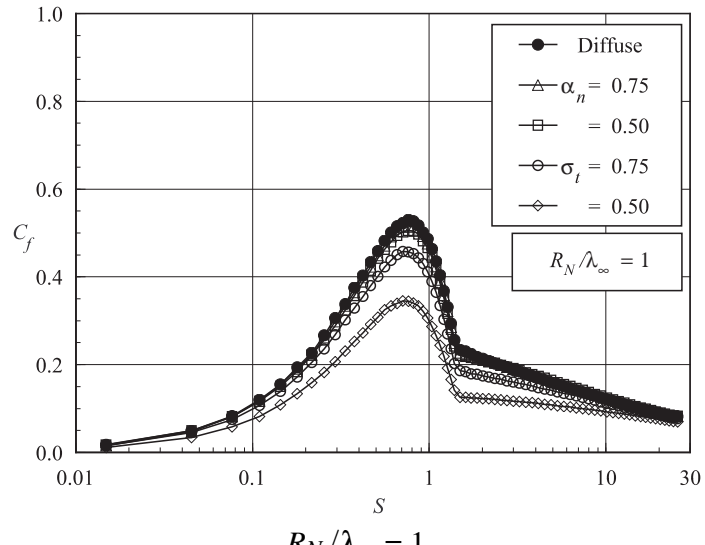

$R_{N} / \lambda_{\infty}=1$

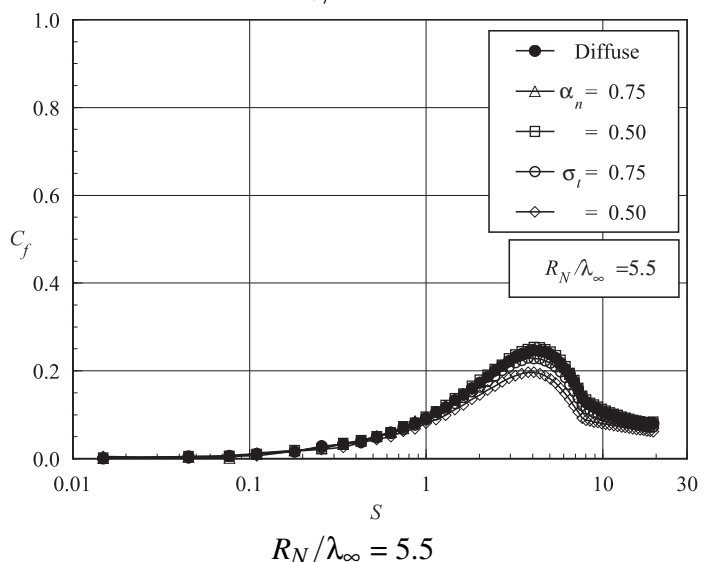

FIG. 8: Distributions of skin friction coefficient $C_{f}$ along the body surface as a function of the accommodation coefficient for round leading edges with $R_{N} / \lambda_{\infty}$ of (a) 0.02 , (b) 1 and (c) 5.5 .

of 5.5, the major contribution to the total drag coefficient is attributed to the pressure, a blunt leading edge characteristic.

In what follows, the effect of changing independently the normal $\alpha_{n}$ and tangential $\sigma_{t}$ accommodation coefficients on the total drag coefficient $C_{d}$ for the round leading edges investigated is tabulated in Table IV.

Referring to Table IV, it can be seen that variations on $\alpha_{n}$ or $\sigma_{t}$ have a different effect on the total drag coefficient $C_{d}$. The total drag coefficient $C_{d}$ increases around $2 \%$ or $3 \%$ by a reduction in the normal accommodation coefficient $\alpha_{n}$, and sig-

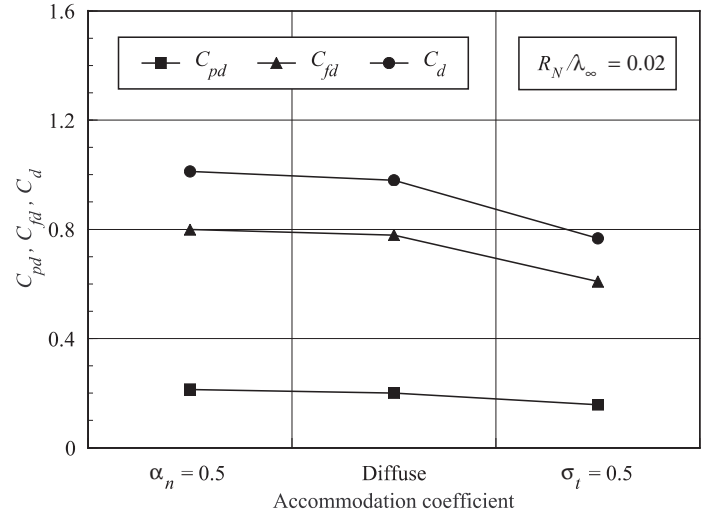

$R_{N} / \lambda_{\infty}=0.02$

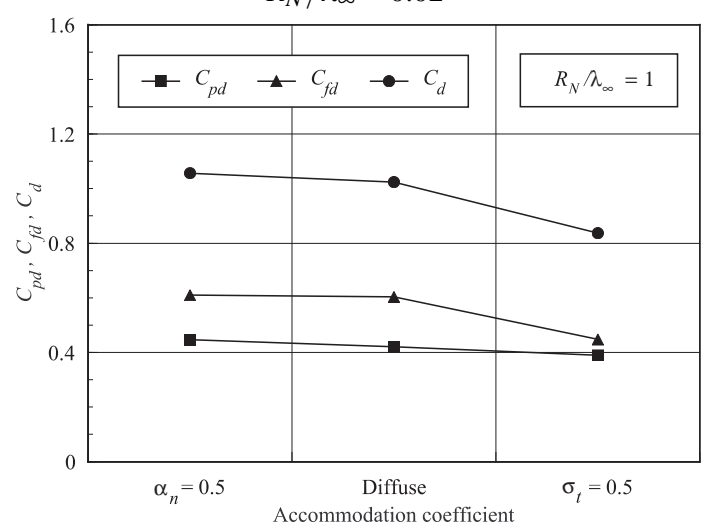

$R_{N} / \lambda_{\infty}=1$

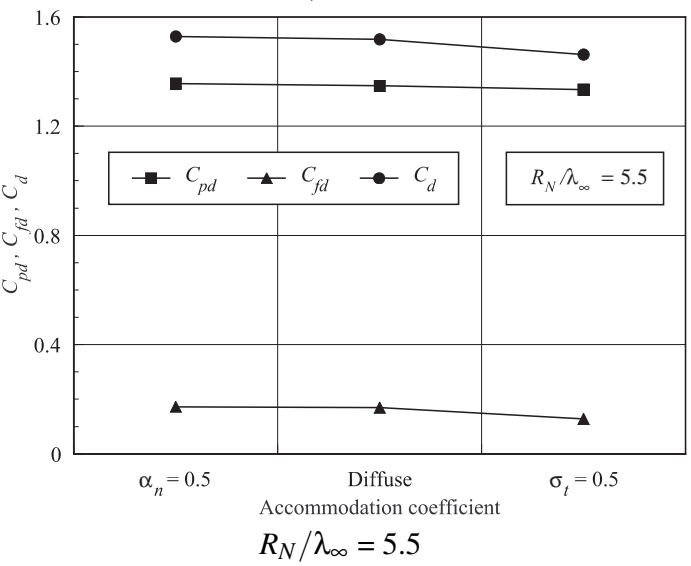

FIG. 9: Pressure drag $C_{p d}$, skin friction drag $C_{f d}$ and total drag coefficient $C_{d}$ as a function of the accommodation coefficient for round leading edges with $R_{N} / \lambda_{\infty}$ of (a) 0.02 , (b) 1 and (c) 5.5 .

nificantly decreases by a reduction in the tangential accommodation coefficient $\sigma_{t}$ for the leading edge shapes investigated. At this point, it should be emphasized that the total drag for round leading edges approaches the wedge drag value with decreasing the nose radius, as would be expected. 
TABLE IV: Total Drag coefficient $C_{d}$ for round leading edges.

\begin{tabular}{cccccc}
\hline \hline$R_{N} / \lambda_{\infty}$ & 0.02 & 0.1 & 1 & 2 & 5.5 \\
\hline Diffuse & 0.978 & 0.979 & 1.028 & 1.085 & 1.519 \\
$\alpha_{n}=0.75, \sigma_{t}=1$ & 0.994 & 0.995 & 1.039 & 1.144 & 1.523 \\
$\alpha_{n}=0.50, \sigma_{t}=1$ & 1.011 & 1.012 & 1.057 & 1.158 & 1.528 \\
$\alpha_{n}=1, \sigma_{t}=0.75$ & 0.908 & 0.910 & 0.957 & 1.074 & 1.496 \\
$\alpha_{n}=1, \sigma_{t}=0.50$ & 0.766 & 0.767 & 0.837 & 0.975 & 1.462 \\
\hline \hline
\end{tabular}

\section{SHOCK-WAVE STANDOFF DISTANCE}

The problem of predicting the shape and location of detached shock waves has been stimulated by the necessity for blunt noses and leading edges configurations designed for hypersonic flight in order to cope with the aerodynamic heating. Also, the ability to predict the shape and location of shock waves is of primary importance in analysis of aerodynamic interference.

In a rarefied flow, the shock wave has a finite region that depends on the transport properties of the gas, and can no longer be considered as a discontinuity obeying the classical Rankine-Hugoniot relations. In this context, the shock standoff distance is defined as being the distance between the shock-wave center and the nose of the leading edge along the stagnation streamline.

In order to quantify the shock standoff distance, the shockwave center (shock-wave location) is determined by employing the following procedure [26]: the flow is assumed to consist of three distinct classes of molecules; those molecules from the freestream that have not been affected by the presence of the leading edge are denoted as class I molecules; those molecules that, at some time in their past history, have struck and been reflected from the body surface are denoted as class II molecules; and those molecules that have been indirectly affected by the presence of the body are defined as class III molecules.

It is assumed that the class I molecule changes to class III molecule when it collides with class II or class III molecule. Class I or class III molecule is progressively transformed into class II molecule when it interacts with the body surface. Also, a class II molecule remains class II regardless of subsequent collisions and interactions. Hence, the transition from class I molecules to class III molecules may represent the shock wave, and the transition from class III to class II defines the boundary layer.

For illustration purpose, the distribution of molecules for each class along the stagnation streamline associated to sharp and blunt leading edges are demonstrated in Figs. 10 and 11, respectively. The impact of the surface accommodation on class I, II and II, related to round leading edge with $R_{N} / \lambda_{\infty}$ of 0.02 is demonstrated in Figs. 10(a-c) for diffuse case, $\alpha_{n}$ of 0.5 (with $\sigma_{t}=1$ ) and $\sigma_{t}$ of 0.5 (with $\alpha_{n}=1$ ), respectively. Similarly, Figs. 11(a-c) display the effect of the surface ac-

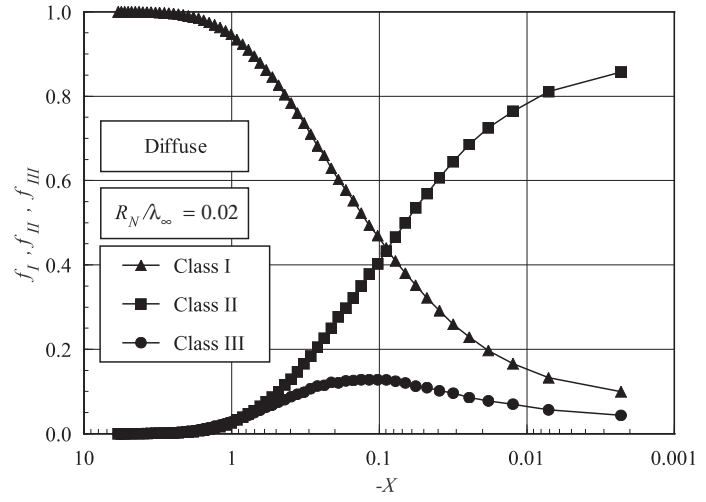

Diffuse

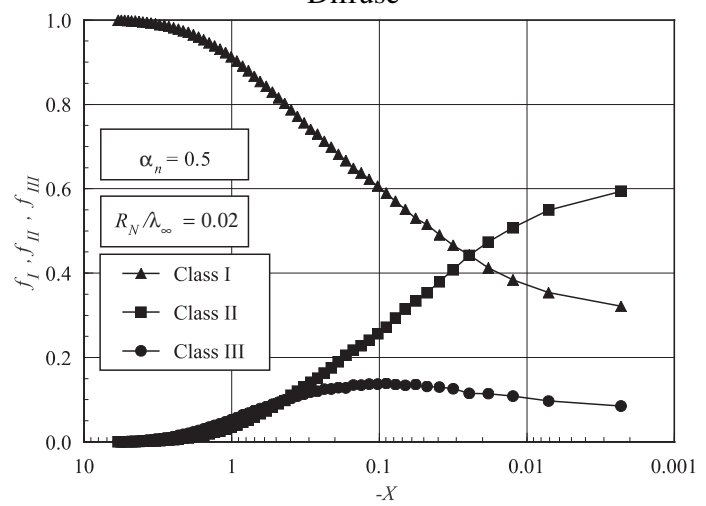

$\alpha_{n}=0.5$ and $\sigma_{t}=1$

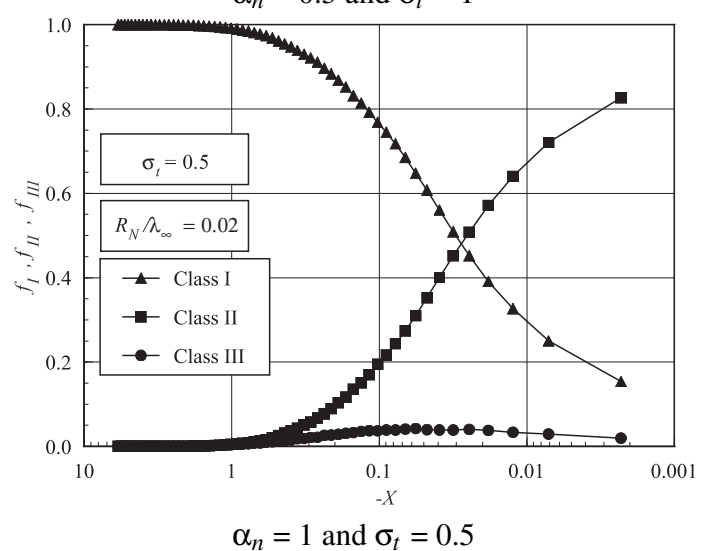

FIG. 10: Distributions of molecules for classes I, II and III along the stagnation streamline for the round leading edge case with $R_{N} / \lambda_{\infty}=$ 0.02: (a) diffuse reflection, (b) $\alpha_{n}=0.5$ and $\sigma_{t}=1$, and (c) $\alpha_{n}=1$ and $\sigma_{t}=0.5$.

commodation for round leading edge with $R_{N} / \lambda_{\infty}$ of 5.5 , the bluntest round leading edge investigated. In this set of figures, $X$ is the distance $x$ along the stagnation streamline (see Fig. 3), normalized by the freestream mean free path $\lambda_{\infty}$, and $f_{I}, f_{I I}$, and $f_{I I I}$ are the number of molecules for classes, I, II and III, respectively, to the total amount of molecules inside each cell. Based on these diagrams, the shock-wave standoff distance is defined as being the distance along the stagnation streamline from the shock wave center, position corresponding to the maximum value for $f_{I I I}$, to the nose of the leading edge. 


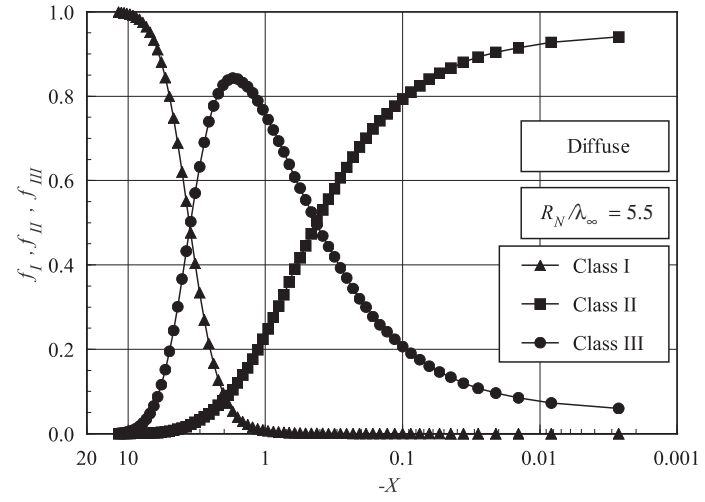

Diffuse

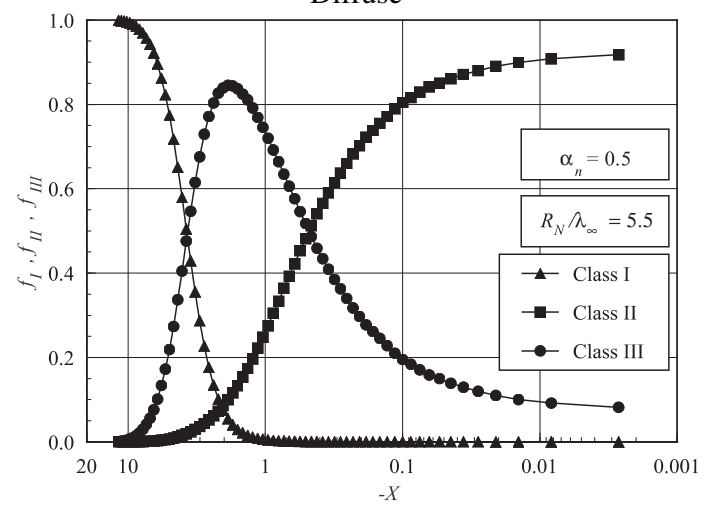

$\alpha_{n}=0.5$ and $\sigma_{t}=1$

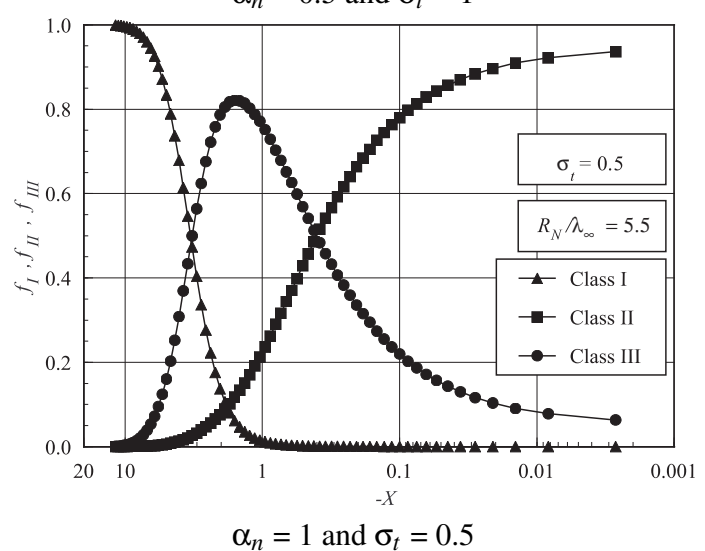

FIG. 11: Distributions of molecules for classes I, II and III along the stagnation streamline for the round leading edge case with $R_{N} / \lambda_{\infty}=$ 5.5: (a) diffuse reflection, (b) $\alpha_{n}=0.5$ and $\sigma_{t}=1$, and (c) $\alpha_{n}=1$ and $\sigma_{t}=0.5$.

By examining Figs. 10 and 11, it is clearly seen that there is a discrete shock standoff distance for the cases shown. It is also seen that partial accommodation coefficient affects the shock-wave standoff distance along the stagnation streamline provided the leading edge is aerodynamically sharp, for instance, $R_{N} / \lambda_{\infty}=0.02$ case. In contrast, no appreciable changes are observed for the bluntest case investigated, $R_{N} / \lambda_{\infty}=5.5$, as either the normal $\alpha_{n}$ or tangential $\sigma_{t}$ accommodation coefficient is reduced from 1 to 0.5 .

Of great significance in Figs. 10 and 11 is the behavior of the class I molecules for sharp and blunt leading edges.

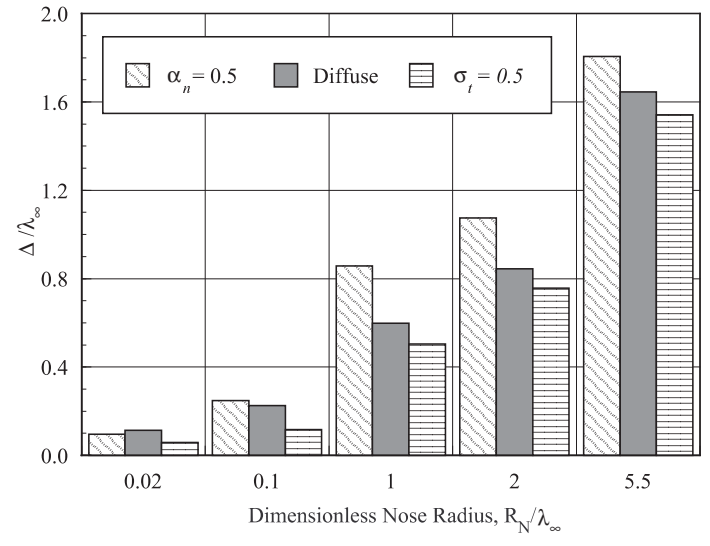

FIG. 12: Dimensionless shock wave standoff distance $\Delta / \lambda_{\infty}$ along the stagnation streamline for round leading edges as a function of the normal and tangential accommodation coefficients.

It should be observed that molecules from freestream, represented by class I molecules, collide with the nose of the leading edges even after the establishment of the steady state. This is shown in Figs. 10, which represent the sharp leading edge case. In contrast, molecules from freestream basically do not reach the nose of the leading edge for those cases illustrated in Figs. 11, which represent blunt leading edges. This is explained by the fact that density increases much more at the vicinity of the stagnation region for blunt leading edges, and reaches its maximum value in the stagnation point. In this connection, the buildup of particle density near the nose of the leading edge acts as a shield for the molecules coming from the undisturbed stream. Consequently, the heat flux at the stagnation point for sharp leading edge is higher than that for blunt leading edge, as shown in Table III.

The impact of changing independently the normal and tangential accommodation coefficients on the shock-wave standoff distance $\Delta$ for the round leading edges investigated is illustrated in Fig. 12, and the values are tabulated in Table V, normalized by the freestream mean free path $\lambda_{\infty}$.

TABLE V: Dimensionless shock wave standoff distance $\Delta / \lambda_{\infty}$ for round leading edges.

\begin{tabular}{cccccc}
\hline \hline$R_{N} / \lambda_{\infty}$ & 0.02 & 0.1 & 1 & 2 & 5.5 \\
\hline Diffuse & 0.114 & 0.226 & 0.598 & 0.845 & 1.645 \\
$\alpha_{n}=0.75, \sigma_{t}=1$ & 0.104 & 0.260 & 0.727 & 0.956 & 1.728 \\
$\alpha_{n}=0.50, \sigma_{t}=1$ & 0.096 & 0.249 & 0.858 & 1.075 & 1.805 \\
$\alpha_{n}=1, \sigma_{t}=0.75$ & 0.074 & 0.159 & 0.551 & 0.818 & 1.620 \\
$\alpha_{n}=1, \sigma_{t}=0.50$ & 0.058 & 0.117 & 0.504 & 0.757 & 1.541 \\
\hline \hline
\end{tabular}

According to Fig. 12 and Table V, there is a discrete shock standoff distance for the round leading edge cases investigated. Furthermore, the shock standoff distance decreases with diminishing the nose radius $R_{N}$. This is an expected result since shock standoff distance on a circular cylinder scales 
with the curvature radius. As a reference, the bluntest leading edge, $R_{N} / \lambda_{\infty}=5.5$ case, presents values for $\Delta / \lambda_{\infty}$ that are one order of magnitude larger than that for the sharpest leading edge investigated, $R_{N} / \lambda_{\infty}=0.02$ case.

It is worthwhile mentioning that the displacement of the shock wave is especially undesirable in a waverider vehicle, because this hypersonic configuration usually depends on shock wave attachment at the leading edge to achieve its high lift-to-drag ratio at high-lift coefficient. Shock wave detachment will allow pressure leakage from the lower surface of the vehicle to the upper surface, thereby degrading the aerodynamic performance of the vehicle.

\section{CONCLUDING REMARKS}

Direct Simulation Monte Carlo method is applied to examine rarefied gas over a group of round leading edges. Effects of incomplete surface accommodation on the heat transfer, total drag and shock-wave standoff distance for a representative range of normal and tangential accommodation coefficients are investigated. The normal and tangential accommodation coefficients are varied from 1.0 to 0.5 , and the nose radius considered in this study covers hypersonic flow from the transition flow regime to the free molecular flow regime.

Calculations showed that a reduction in the normal accommodation coefficient from 1.0 to 0.5 decreased the heat trans- fer coefficient at the vicinity of the stagnation point for the round shapes investigated. In contrast, a reduction in the tangential accommodation coefficient increased slightly the heat transfer coefficient near the nose of the leading edges. Also, it was found that the total drag coefficient is reduced by a reduction in the tangential accommodation coefficient, and increased by a reduction in the normal accommodation coefficient.

The analysis also showed that the shock standoff distance is sensitive to changes on the surface accommodation coefficient. In general, it was observed that shock wave standoff distance decreased by a reduction on the tangential accommodation coefficient and increased with reducing the normal accommodation coefficient.

The effects of either normal or tangential accommodation coefficient showed that in order to make accurate predictions of the aerodynamic forces on, and heat transfer rates to, bodies in rarefied hypersonic flow it will be necessary to take surface accommodation into account.

The calculations presented in this work have only covered a limited number of parametric variations. Further calculations with additional combinations of normal and tangential accommodation coefficients or where the internal energy accommodation is varied independently might provide more insight into the sensitivity of the aerothermodynamic quantities to gas-surface model.
[1] T. R. F. Nonweiler, J. Roy. Aero. Soc., 63, 521 (1959).

[2] J. L. Anderson, in Proceedings of the 1st International Hypersonic Waverider Symposium, Univ. of Maryland, College Park, MD, 1990.

[3] J. L. Potter and J. K. Rockaway, in Rarefied gas Dynamics: Space Science and Engineering, edited by B. D. Shizgal and D. P. Weaver, Progress in Astronautics and Aeronautics, AIAA New York, 160, 296 (1994).

[4] D. F. G. Rault, J. Space. Rockets, 31, 719 (1994).

[5] R. E. Graves and B. M. Argrow, in 35th AIAA Thermophysics Conference, AIAA Paper 2001-2960, Anaheim, CA, 2001.

[6] A. I. Shvets, V. I. Voronin, I. M. Blankson, V. Khikine, and L. Thomas, in 43rd AIAA Aerospace Sciences Meeting and Exhibit, AIAA Paper 2005-0512, Reno, NV, 2005.

[7] R. G. Lord, in 17th International Symposium on Rarefied Gas Dynamics, edited by A. E. Beylich, Aachen, Germany, 1991.

[8] C. Cercignani and M. Lampis, Trans. Theor. Stat. Phys., 1, 101 (1971).

[9] R. G. Lord, Phys. Fluids, A 3, 706 (1991).

[10] R. S. Simmons and R. G. Lord, in 19th International Symposium on Rarefied Gas Dynamics, edited by J. Harvey and G. R. Lord, Oxford, 1994.

[11] R. G. Lord, Phys. Fluids, 7, 1159 (1995).

[12] G. A. Bird, Molecular Gas Dynamics and the Direct Simulation of Gas Flows, Oxford University Press, Oxford, England, UK, 1994.

[13] C. Cercignani, Trans. Theor. Stat. Phys., 72, 27 (1972).
[14] F. Sharipov, J. Fluid Mech., 518, 35 (2004).

[15] M. S. Woronowicz and D. F. G. Rault, J. Space. Rockets, 31, 532 (1994)

[16] M. O. Hedahl and R. G. Wilmoth, NASA TM-110205, 1995

[17] C. Cercignani, The Boltzmann Equation and Its Applications, Springer-Verlag, New York, NY, 1988.

[18] A. L. Garcia and W. Wagner, Phys. Fluids, 12, 2621 (2000).

[19] N. G. Hadjiconstantinou, Phys. Fluids, 12, 2634 (2000).

[20] G. A. Bird, in Progress in Astronautics and Aeronautics: Rarefied gas Dynamics, edited by Sam S. Fisher, AIAA New York, 74, 239 (1981).

[21] G. A. Bird, in Rarefied gas Dynamics: Theoretical and Computational Techniques, edited by E. P. Muntz, D. P. Weaver, and D. H. Campbell, Progress in Astronautics and Aeronautics, AIAA, New York, 118, 374 (1989).

[22] C. Borgnakke and P. S. Larsen, J. Comp. Phys., 18, 405 (1975).

[23] F. J. Alexander, A. L. Garcia, and B. J.Alder, Phys. Fluids, 10, 1540 (1998).

[24] F. J. Alexander, A. L. Garcia, and B. J.Alder, Phys. Fluids, 12, 731 (2000).

[25] K. Guo and G.-S. Liaw, in Proceedings of the 35th AIAA Thermophysics Conference, AIAA Paper 2001-2953, Anaheim, CA, 2001.

[26] W. F. N. Santos, in Proceedings of the 24th International Congress of the Aeronautical Sciences, ICAS 2004, Yokohama, Japan, 2004. 